\title{
HUMUS DE LOMBRICULTURA PROVENIENTE DE DIFERENTES INSUMOS ORGANICOS Y SU EFECTO EN EL RENDIMIENTO DE PEPINO EN UN ULTISOL DEGRADADO DE PUCALLPA
}

Olga Ríos del Aguila *

Paco Rivera Gonzales **

\section{RESUMEN}

En Pucallpa-Perú se realizó un experimento para evaluar: 1) el contenido de nutrientes de humus de lombricultura (HL) proveniente de ocho mezclas de insumos orgánicos tales como estiércol de vacuno más aserrín, estiércol de ovino más aserrín, sólo estiércol de vacuno, sólo estiércol de ovino, estiércol de vacuno más residuos de cervecería, estiércol de ovino más residuos de cervecería, estiércol de vacuno más malezas, estiércol de ovino más malezas, y ¡1) evaluar el efecto de HL de diferentes mezclas en el rendimiento de pepino, en un ultisol degradado de Pucallpa.

El mayor contenido de $\mathrm{N}(2.6 \%)$ se encuentra en el HL proveniente de la mezcla de ovino + residuos de cervecería, y los mayores contenidos de $\mathrm{P}$ $(0.34 \%), \mathrm{K}(0.003 \mathrm{ppm}), \mathrm{Ca}(1.27 \%)$ y $\mathrm{Mg}(3.92 \%)$, en el HL proveniente del estiércol de ovino.

La calidad agronómica de los diferentes HL se experimentó en el cultivo de pepinillo (Cucumis .sativus) var. "Palomar", en el número (190) y peso (75 $\mathrm{kg}$ ) de frutos por $10 \mathrm{~m}^{2}$, se obtuvo por efecto de HL proveniente de la mezcla ovino + residuo de cervecería, lo cual fue consistente con el mayor contenido de $\mathrm{N}, \mathrm{P}, \mathrm{K}, \mathrm{Ca}$ y $\mathrm{Mg}$ presente en este HL.

\section{INTRODUCCION}

Parte de los suelos degradados de la región Ucayali pueden usarse con cultivos exigentes en nutrientes como hortalizas, mediante la aplicación de abonos orgánicos tales como HL. Las limitaciones al uso de los suelos degradados en la región Ucayali se debe en gran parte, a su mal manejo, los cuales en diferentes estados de degradación presentan severa deficiencia de nutrientes y problemas en

\footnotetext{
* Profesora asociada de la UNU, especialista en suelos; Investigadora IIAP

** Tesista de la UNU
} 
sus propiedades físicas, principalmente compactación del suelo (Ara, 1992; Rime, 1992).

En Pucallpa, parte de los suelos degradados pueden ser recuperados mediante un manejo adecuado con incorporación de materia orgánica, como el HL, en la producción de hortalizas (Rime y Ríos 1992 Ríos et al., 1993). La lombricultura es una actividad que está siendo practicada en la costa y sierra peruanas, el IIAP-Ucayali (Salas, 1989) lo hace en el trópico húmedo.

Probablemente la calidad del HL depende de la calidad del alimento que ingieren las lombrices en pasturas mejoradas de alto rendimiento, o que reciben suplementos. En Ucayali existen diversos insumos orgánicos disponibles que pueden ser aprovechados para obtener abonos orgánicos de calidad.

En base a estas consideraciones, este trabajo tuvo como objetivo 1) determinar el contenido de nutrientes de los HL provenientes de diferentes insumos alimenticios que ingiere la lombriz, y ii) evaluar la calidad agronómica en términos de rendimiento de peso y número de frutos de pepino.

\section{REVISION BIBLIOGRAFICA}

\section{SUELOS DE LE SELVA PERUANA}

En la selva peruana predominan los ultisoles, entisoles, alfisoles, y los inceptisoles. Los primeros son ácidos, rojos y amarillos, de baja fertilidad; predominan en terrenos de altura de la selva baja, así como en terrazas antiguas como Pucallpa (ONERN, 1979).

Los factores edáficos más limitantes de los suelos de la Amazonía Peruana son más de orden químico que físico, siendo más abundante la deficiencia de materia orgánica, $\mathrm{N}(94 \%), \mathrm{P}(66 \%)$, y bajas reservas de $\mathrm{K}, \mathrm{Mg}$ y otros nutrimentos $(64 \%)$, el porcentaje de saturación de $\mathrm{Al}(65 \%)$ aumenta a medida que aumenta la profundidad, sucede lo contrario con el porcentaje de saturación de bases que disminuye en cuanto aumenta la profundidad. Dos importantes limitaciones químicas típicas de los trópicos se manifiestan en los suelos de la zona: 1) baja capacidad de intercambio catiónico, lo cual favorece la lixiviación de los elementos; 2) capacidad relativamente alta de fijar fertilizantes fosfatados en forma poco disponible (Sánchez y Benites, 1983).

La adición de materia orgánica a suelos ácidos y deficientes en nutrientes es importante para elevar el contenido de macronutrientes y micronutrientes. Ella activa los procesos microbiales, mejorando las propiedades físicas y químicas, regula la temperatura, retarda la fijación del $\mathrm{P}$, y favorece el crecimiento y desarrollo de los cultivos (Jacobs y Uexhull, 1966; Monroy y Viniegra 1981; Gros, 1981). 


\section{HUMUS DE LOMBRICULTURA}

El HL es el proceso final de la descomposición de la materia orgánica, realizado por lombrices. Ellas mineralizan enzimáticamente la materia orgánica en el primer tercio de su aparato digestivo y luego la humifican en la parte posterior del intestino por acción de los microorganismos presentes en esta sección intestinal. La cantidad N del HL depende de la alimentación que consume la lombriz (SABAC-Chile, 1987, Banco Agrario, 1987).

El HL es una materia orgánica granulosa, inodora de color café oscuro. El proceso de degradación de la materia orgánica digerida por las lombrices se realiza en forma acelerada (hora/día) si se compara con el proceso de descomposición natural. El HL es un producto químicamente estable (Banco Agrario, 1987).

El HL está compuesto de N,P, k, Ca, Mg y oligoelementos, tales como $\mathrm{Fe}, \mathrm{Cu}, \mathrm{Zn}, \mathrm{Mn}$ y Bo. Aligera los terrenos arcillosos y agrega a los arenosos. Por ser un coloide tiene una retención de humedad de 16 veces su peso en agua. Impide la lixiviación del $\mathrm{N}, \mathrm{K}$, y la insolubilización del $\mathrm{P}$. Intensifica las características y los valores de los fertilizantes químicos al ser usados en asociación con éstos ahorrando hasta un 25\% (SABAC-Chile, 1987).

Mediante el uso del HL se logra un aumento significativo de la productividad de los suelos degradados de Pucallpa, aplicado ya sea en forma total o localizada, especialmente en frutales, hortalizas y plantas ornamentales (Ríos y Rime, 1992; Ríos et al., 1993).

El HL se presenta como una alternativa ecológica para la 'degradación de todo tipo de desecho biodegradable (Ferrruzi, 1987). En el IIAP-Ucayali, Sánchez (1990) informó que el HL proveniente de una mezcla de guano de vacuno + paja de malezas tuvo un $\mathrm{pH} 45,7,1.20 \%$ de N, $0.95 \%$ de $\mathrm{P}, \mathrm{y} 0.47 \%$ de K. Este HL fue probado en un suelo degradado con siembras de ají dulce (Capsicum annum). Las dosis estudiadas fueron $0,0.250,0.500,0.750,1.0 \mathrm{~kg}$ $\mathrm{HL} /$ planta.

Se encontró que con dosis de $1.0 \mathrm{~kg} \mathrm{HL} /$ planta en poepino se obtuvo un rendimiento de $35 \mathrm{~kg}$ de fruto fresco por $10 \mathrm{~m} 2$ (Ríos et al., 1993).

\section{MATERIALES Y MÉTODOS}

\section{LOCALIZACIÓN DEL ESTUDIO}

El estudio se realizó en un ultisol degradado de Pucallppa, en el margen derecho del Km. 12.4 de la carretera Federico Basadre. La zona corresponde al 
Bosque Estacional Semisiempre-verde. Las coordenadas geográficas son 8²2'31' S, 74³4'35' O. La altitud es 145 msnm.

El estudio se realizó de abril a octubre de 1990, se ejecutó en dos etapas; obtención de HL, y su evaluación agronómica en el rendimiento del pepino.

\section{OBTENCIÓN DEL HUMUS DE LOMBRICULTURA}

Para la obtención del HL se ensayó las siguientes mezclas de alimento para la lombriz; estiércol de vacuno más aserrín, estiércol de ovino más aserrín, sólo estiércol de vacuno, sólo estiércol de ovino, estiércol de vacuno más orujo (residuos de cervecería), estiércol de ovino más orujo, estiércol de vacuno más malezas, estiércol de ovino más malezas. La preparación de la mezcla se hizo en una relación 70:30 de estiércol y residuo vegetal respectivamente, en base a peso seco.

Se siguió los procesos de fermentación de la mezcla establecidos por el IIAP-Ucayali, según Salas (1989) y Sánchez (1990). El tiempo de descomposición varió de acuerdo al tipo de mezcla y fue entre 20 a 36 días. Estos se incorporaron al lecho para alimentar a las lombrices. La inoculación fue de 1000 lombrices $/ \mathrm{m}^{2}$. Cuando el HL tuvo las características deseadas se cosechó y se analizó el contenido de nutrientes.

\section{EVALUACIÓN DEL HL EN EL RENDIMIENTO DE PEPINO}

\section{Condiciones climáticas y de suelo}

El clima es tropical, con una temperatura media mensual de $25^{\circ} \mathrm{C}$, con $77 \%$ de humedad relativa. La precipitación pluvial anual es $1354 \mathrm{~mm}$ a $6 \mathrm{Km}$. de Pucallpa.

\section{Propiedades de suelo del área experimental}

Las propiedades del suelo $(0.20 \mathrm{~cm})$ se presenta en el cuadro 1 . El suelo es ácido ( $\mathrm{pH} 4.5$ ), con $1.75 \%$ de materia orgánica, y $2.9 \mathrm{ppm}$ de P-Olsen. Los niveles de los cationes cambiables son de 1.6 meq $100 \mathrm{~g}^{-1}$ de $\mathrm{Ca}+\mathrm{Mg}$, y 1.4 meq $100 \mathrm{~g}^{-1}$ de $\mathrm{Al}$. La saturación de $\mathrm{Al}$ es de $38 \%$. El suelo es franco. 


\section{Cuadro 1}

\section{PROPIEDADES DEL SUELO DEL AREA EXPERIMENTAL}

\begin{tabular}{|c|c|c|c|c|c|c|}
\hline Arena Arcilla & $\mathrm{pH}$ & MO & N & $\begin{array}{l}\mathrm{P} \\
\mathrm{pm}\end{array}$ & $\begin{array}{l}\mathrm{Ca}+\mathrm{Mg} \\
--- \text { meq }\end{array}$ & $\begin{array}{l}\mathrm{Al} \mathrm{Al} \\
\%\end{array}$ \\
\hline $34 \quad 24$ & 4.6 & 1.8 & 0.081 & 2.6 & 1.5 & 1.337 .1 \\
\hline II $\quad 32 \quad 28$ & 4.5 & 1.7 & 0.076 & 2.6 & 1.6 & 1.640 .0 \\
\hline III $36 \quad 24$ & 4.6 & 1.7 & 0.076 & 3.4 & 1.7 & 1.335 .1 \\
\hline
\end{tabular}

I, II, III $=$ repeticiones

Variable de respuesta: rendimiento de pepino

Para ver el efecto de los HL proveniente de diferentes mezclas se usó los rendimientos en peso y número de frutos de pepino. Las evaluaciones se las realizó a los 51, 53 y 56 días después de la siembra.

\section{Diseño y análisis estadístico}

Se usó el diseño de Bloques Completos al Azar con 27 unidades experimentales, generado por 9 tratamientos (ocho tipos de HL más un testigo sin aplicación) y 3 repeticiones.

\section{Preparación del suelo y aplicación del HL}

La preparación del suelo fue mecanizado con arado de discos una profundidad de $15 \mathrm{~cm}$. a los 5 días se realizó dos pasadas de rastra cruzada.

Las parcelas de $1 \mathrm{~m} \times 10 \mathrm{~m}$ se las levantó a $0.30 \mathrm{~m}$ sobre el nivel del suelo. Se incorporó HL en forma localizada en dosis de $1 \mathrm{~kg} /$ planta en base a peso seco (el HL tuvo un $50 \%$ de humedad). Se sembró el pepino var. "Palomar" con 3 semillas por golpe, $0.80 \mathrm{~m}$. entre plantas en la parte central de la parcela, obteniéndose 12 sitios para las plantas. Cuando la planta tuvo $15 \mathrm{~cm}$ 
se eliminó una planta quedando dos plantas por golpe, y se aporcó. Los riegos fueron cada 3 a 4 días hasta la madurez. El control de malezas fue manual.

\section{RESULTADOS Y DISCUSION}

\section{CONTENIDO DE NUTRIENTES DE HL}

En el cuadro 2 se presenta el análisis químico del HL proveniente de diferentes alimentos. En vista que no hubo repeticiones en el análisis no podemos hacer generalizaciones. Sin embargo, de manera puntual podemos decir que el contenido de $\mathrm{N}$ fue mayor en el HL proveniente de la mezcla de ovino \pm residuo de cervecería con $2.6 \%$, seguido de ovino + maleza con $2.2 \%$, ya que casi duplicaron a los demás HL procedentes de diferentes mezclas.

En el contenido de $\mathrm{P}$ los niveles más altos fueron para el estiércol sólo de ovino (0.35\$), superando en dos veces a los demás HL; pero vacuno + aserrín tuvo el menor valor $(0.099 \%)$. En K hubo mayor contenido en el de sólo de ovino $(0.003 \mathrm{Ppm})$, y en vacuno + maleza $(0.00225 \mathrm{ppm})$, también fueron el doble de $\mathrm{K}$ en comparación a los demás HL. Para, Ca se encontró mayores contenidos en HL proveniente de estiércol sólo de ovino y ovino + aserrín, con $1.28 \%$ para ambos, seguido el de sólo de vacuno y ovino + maleza $(0.72 \%)$. En el contenido de $\mathrm{Mg}$, los niveles más altos fueron para el de sólo de ovino (3.92\%), ovino más orujo (2.24\%), y ovino + maleza (1.76\%) (cuadro 2).

\section{Cuadro 2}

\section{ALGUNAS PROPIEDADES QUÍMICAS Y CONTENIOD DE NUTRIENTES DE HUMUS DE LOMBRICULTURA (HL) PROVENIENTE DE DIFERENTES MEZCLAS OBTENIDAS EN PUCALLPA-PERÚ, 1991}

\begin{tabular}{|c|c|c|c|c|c|c|c|c|}
\hline $\begin{array}{l}\text { HL según } \\
\text { mezclas }\end{array}$ & $\mathrm{pH}$ & Mo & $\mathrm{C} / \mathrm{N}$ & $\mathrm{N}$ & $\mathrm{P}$ & $\mathrm{K}$ & $\mathrm{Ca}$ & $\mathrm{Mg}$ \\
\hline $\mathrm{Va}$ & 6.7 & 75.59 & 39.49 & 1.11 & 0.19 & 0.00195 & 1.04 & 1.44 \\
\hline Ov & 5.2 & 76.10 & 40.12 & 1.10 & 0.34 & 0.003 & 1.28 & 3.92 \\
\hline $\mathrm{Va}+\mathrm{As}$ & 6.1 & 68.07 & 34.63 & 1.14 & 0.099 & 0.0012 & 0.32 & 1.28 \\
\hline $\mathrm{Ov}+\mathrm{As} 6.6$ & 56.07 & 19.71 & 1.65 & 0.14 & 0.0015 & 1.28 & 1.04 & \\
\hline $\mathrm{Va}+\mathrm{Or}$ & 6.2 & 66.72 & 29.32 & 1.31 & 0.16 & 0.0011 & 0.32 & 1.6 \\
\hline $\mathrm{Ov}+\mathrm{Or} 6.5$ & 58.37 & 13.04 & 2.60 & 0.13 & 0.0017 & 0.24 & 2.24 & \\
\hline $\mathrm{Va}+\mathrm{Ma} 6.0$ & 70.10 & 34.45 & 1.18 & 0.13 & 0.0012 & 0.40 & 1.44 & \\
\hline $\mathrm{Va}+\mathrm{Ma} 7.6$ & 61.12 & 15.56 & 2.27 & 0.12 & 0.00225 & 0.72 & 1.76 & \\
\hline
\end{tabular}

Estiércol de $\mathrm{Va}=$ vacuno, estiércol de $\mathrm{Ov}=$ ovino, As $=$ aserrín, Or $=$ orujo, $\mathrm{Ma}=$ maleza. 
En resumen, los HL provenientes de residuos de estiércol sólo de ovino y en mezcla tuvieron los mayores contenidos de $\mathrm{N}, \mathrm{P}$, , K, Ca, y Mg. Esto puede deberse a que el estiércol de ovino fue colectado de un aprisco con techo, fue un guano viejo y de hecho Muyo casi toda la orina del animal. Probablemente estas condiciones hacen que el guano sea más rico en nutrientes (Worthen y Aldrich, 1980; Ensminger, 1976; Flores, 1986). Flores (1986) señala que se pierde muchos nutrientes del guano si la orina ocurre fuera del establo. La orina del ganado vacuno puede contener hasta la mitad del $\mathrm{N}$ excretado, y tres cuartas partes del K. Casi todo el $\mathrm{Ca}$ y el $\mathrm{P}$ se encuentra en las heces. Por tanto, el mayor contenido de nutrientes en $\sim$ ¡IL, proveniente de guano de ovino o sus mezclas, son consistentes

estas aseveraciones.

Por otra parte, Carrión (1990) realizó un trabajo similar al nuestro en Tingo María, pero usando otros recursos vegetales tales como pasto elefante, hojas de bambú, panca de maíz y cascarilla de cacao, mezcladas con guano de vacuno. Los valores del contenido de $\mathrm{N}$ estuvieron en un rango de 2.58 a $3.30 \%$, a $2.0 \%$ de $\mathrm{P}$, de 3.60 a 425 de $\mathrm{Ca}+\mathrm{Mg}$. Estos valores son ligeramente superiores a los obtenidos en el presente trabajo. Este efecto puede deberse al tipo de insumo para la obtención del HL.

\section{EVALUACIÓN DEL HL EN EL RENDIMIENTO DEL PEPINO}

En general, los mayores rendimientos de peso fresco de pepino se encontraron por efecto de HL proveniente de ovino, ovino + maleza, ovino + aserrín, y ovino + orujo. Las mezclas con guano de vacuno tuvieron menores rendimientos (gráfico 1).

En el cuadro 3 muestra los análisis de varianza del rendimiento en peso y número de frutos de pepino. Existe diferencias significativas en el rendimiento de peso de frutos $(\mathrm{p}<0.01)$ y en el número de frutos $(\mathrm{p}<0.01)$, por efecto de la aplicación de los HL proveniente de diferentes insumos orgánicos. Asimismo, existe diferencia estadística $(\mathrm{p}<0.05)$ entre bloques, lo que sugiere que el diseño de bloques completos al azar fue eficiente. 


\section{Cuadro 3}

CUADRADOS MEDIOS Y SIGNIFICACIONES DEL ANÁLISIS DE VARIANZA DEL RENDIMIENTO EN PESO Y NÚMERO DE FRUTOS DE PEPINO.

\begin{tabular}{llll}
\hline FV & GL & Peso de frutos & $\mathrm{N}^{\mathrm{o}}$ de frutos \\
\hline Bloques & 2 & $504.0 *$ & $5,186.1^{*}$ \\
Tratamiento & 8 & $2,370.2 * *$ & $8,717.8^{* *}$ \\
Error & 16 & 108.4 & 825.9 \\
\hline $\mathrm{CV}$ & $21 \%$ & $22 \%$ \\
\hline$* * *$ & $=$ Significativo a un nivel de probabilidad de 0.05 y 0.01, respectivamente. \\
$\mathrm{CV}$ & $=$ &
\end{tabular}

En términos de la Prueba de Duncan $(\mathrm{p}<0.05)$ el HL proveniente de la mezcla ovino + orujo tuvo el mayor rendimiento en peso de frutos $(75 \mathrm{~kg})$ por cada $10 \mathrm{~m}^{2}$ (cuadro 4). Ovino + maleza, sólo ovino, ovino + aserrín y sólo vacuno no presentaron diferencia estadística entre sí, con rendimientos de 66.67, $59.33,55.33$ y $55.33 \mathrm{~kg} 10 \mathrm{~m}^{2}$.

\section{Cuadro 4}

RENDIMIENTO EN PESO Y NÚMERO DE FRUTO DE PEPINO POR EFECTO DE HUMUS DE LOMBRICULTURA (HL) PROVENIENTE DE DIFERENTES MEZCLAS, EN UN ULTISOL DEGRADADO DE PUCALLPA-PERÚ, PROMEDIO DE 3 REPETICIONES, 1991.

\begin{tabular}{lllll}
\hline HL según mezclas & \multicolumn{2}{l}{$\begin{array}{l}\text { Rendimiento } \\
\text { Peso } \\
\text { kg } 10 \mathrm{~m}^{2}\end{array}$} & \multicolumn{2}{l}{$\begin{array}{l}\text { Número de frutos } \\
\text { Unidades }\end{array}$} \\
\hline Ovino + orujo & $75.0 \mathrm{a}$ & 190 & $\mathrm{a}$ \\
Ovino + maleza & 66.67 & $\mathrm{ab}$ & 166 & $\mathrm{ab}$ \\
Ovino & $59.33 \mathrm{abc}$ & 153 & $\mathrm{ab}$ \\
Ovino + aserrín & 55.33 & $\mathrm{abc}$ & 150 & $\mathrm{ab}$ \\
Vacuno & 55.33 & $\mathrm{abc}$ & 141 & $\mathrm{ab}$ \\
Vacuno + orujo & 51.33 & $\mathrm{be}$ & 125 & $\mathrm{~b}$ \\
Vacuno + maleza & 47.47 & $\mathrm{be}$ & 119 & $\mathrm{~b}$ \\
Vacuno + aserrín & 40.0 & $\mathrm{e}$ & 0 & $\mathrm{c}$ \\
Testgo sin HL & 00 & $\mathrm{~d}$ & &
\end{tabular}

Letras iguales en la misma columna no son estadísticamente diferentes, según la Prueba de Duncan $(\mathrm{p}<0.05)$. 
Estos superaron a los HL provenientes de vacuno + orujo, 'ieuno + maleza, y vacuno + aserrín, con rendimientos de $51.3,47,67$, y $40.0 \mathrm{~kg}$. de frutos $10 \mathrm{~m} 2$. El testigo sin HL no tuvo rendimiento (cuadro 4). La Prueba de Duncan $(\mathrm{p}<0.05)$ para el número de frutos tuvo un patrón similar al peso e frutos de pepino.

En un trabajo para ver el efecto de dosis de HL en el rendimiento de pepino, Ríos, Calle y Rime (1993) encontraron una estrecha relación lineal y positiva entre el peso de pepino y el número de frutos.

Los mayores rendimiento en peso de frutos de pepino por efecto de los HL proveniente de ovino o sus respectivas mezclas, en $\sim$ rango de 55.3 a $75.0 \mathrm{~kg} 10$ $\mathrm{m} 2$, son consistentes con los mayores niveles de $\mathrm{N} ; \mathrm{P}, \mathrm{K}, \mathrm{Ca}$ y $\mathrm{Mg}$, encontrados en la composición química de los HL.

Trabajos similares acerca del efecto del HL en el rendimiento de pepino, realizado por Ríos et al. (1993), reportan un rendimiento de $34.65 \mathrm{~kg} 10 \mathrm{~m}^{2}$ de frutos de pepino var. "Palomar" con $1 \mathrm{~kg} \mathrm{HL} /$ planta (en base a peso seco) proveniente de una mezcla de vacuno + maleza. El testigo sin HL tampoco tuvo rendimiento.

Los rendimientos del pepino en el presente experimento fueron superiores a los obtenidos por Ríos et al (1993), Bañados (1973), Becerra (1977), Ferrari (1963), Pérez (1989) y Peláez (1990), con fertilización orgánica e inorgánica.

\section{CONCLUSIONES}

En base a los resultados del presente trabajo se ha encontrado que: i) Los mayores niveles de $\mathrm{N}, \mathrm{P}, \mathrm{K}, \mathrm{Ca}$ y $\mathrm{Mg}$, están en los HL provenientes de guano de ovino con mezcla de orujo, maleza y aserrín, ii) La mejor respuesta a la incorporación de HL en el suelo en términos de peso y número de frutos de pepino fue con el HL proveniente de guano de ovino o en mezclas con aserrín, orujo y maleza. Esto fue consistente con su mayor nivel de nutrientes. iii) En suelo sin incorporación de HL no hubo rendimiento.

\section{BIBLIOGRAFIA}

ARA, M.A. 1992. "Relaciones suelo-planta-animal en el ecosistema amazónico". En Foro Internacional sobre Desarrollo Agrario Integral y Manejo Racional de la Amazonía. Pucallpa, 7 al 10 diciembre de 1992. INIAA-Proyecto Suelos Tropicales. (en prensa) 
BANCO AGRARIO DEL PERU. 1987. Manual de instrucciones para la Lombricultura. Departamento de divulgación técnica. Lima. 25 p.

BAÑADOS, F. 1973. "Comparativo preliminar de 6 variedades de 8 Pepinillo (Cucumis sativus, L), para consumo fresco en la zona de Tingo María”. Tesis Ing. Agrón. Universidad Nacional Agraria de la Selva-Tingo María. $68 \mathrm{p}$.

BECERRA, J. 1977. Horticultura. Universidad Nacional Agraria La Molina. Facultad de Agronomía. Departamento de Public. Lima, Perú. 181 p.

ENSMINGER, M. 1976. Producción Ovina. 2da. Ed. Edit. 1 Ateneo. Buenos Aires. 545 p.

FERRERI, G. 1963. "Ensayo comparativo de 5 variedades de pepino para encurtido". Tesis Ing. Agrón. Universidad Nacional Agraria-La Molina. $19 \mathrm{p}$.

FLORES, 1. 1986. Manual de alimentación animal. 1ra. Ed. Ediciones Ciencia y Técnica, S.A. México. 1905 p.

FERRUZI, C. 1987. Manual de Lombricultura. Ed. Mundiprensa. Madrid. 130 p.

GROSS, A. 1981. Abonos. Guía práctica de la fertilización 7ma. Ed. Ediciones Mundi-Prensa. España. 559 p.

JACOBS, H. y U. UEXHULL. 1986. Fertilización: Nutrición y abonado de los cultivos tropicales y sub-tropicales. 2da. ed., Hannover, Verlaggessellschaft for Ackeertban N,B. 626 p.

MONROY, H. y G. VINJEGRA. 1981. Biotecnología para el aprovechamiento de los desperdicios orgánicos. Mexico. 260 p.

ONERN. 1979. Inventario, evaluación e integración de los recursos naturales de la zona Pucallpa-Abujao. Lima, Perú. 225 p.

PELAEZ, E. 1990. "Distanciamiento y niveles de nitrógeno en el cultivo de pepinillo (Cucumis sativus)". Tesis Ing. Agrón. Universidad Nacional Agraria La Selva. Tingo María. 76 p. 
PEREZ, F. 1989. "Efectos de la ceniza de madera y dolomita en el cultivo de hortalizas", Universidad Nacional de Ucayali. Facultad de Ciencias Agropecuarios, Universidad Nacional de Ucayali. Pucallpa-Perú. 60 p

RIME, R. 1992. "Adecuación de secuencia metodológica para estimar la distribución radicular de leguminosas forrajeras en un suelo de Pucallpa". Facultad de Ciencias Agropecuarias, Universidad Nacional de Ucayali. Pucallpa-Perú. 60 p.

RIOS, O. Z. y R. RIME. 1992. "Informe del Proyecto Hortalizas. Instituto de Investigaciones de la Amazonía Peruana-Ucayali Pucallpa. 10 p. (mimeografiado).

RIOS, O.Z., C.A. CALLE, y R. RIME. 1993. "Humus de lombricultura y su efecto en el rendimiento de pepino (Cucumis sativa), ají dulce (Capsicum annun), y chiclayo verdura (Vignia sinensis), en un suelo degradado de Pucallpa”. Instituto de Investigaciones de la Amazonía Peruana-Ucayali. 12 p. (Artículo científico en prensa).

SABAC-CHILLE. 1987. "Lombricultura un amplio horizonte". Centro de desarrollo de Lombricultura SABAC-CHILE. Chile. 52 p.

SALAS, S. 1989. "Informe técnico sobre investigaciones de lombricultura en Trópico". Instituto de Investigaciones de la Amazonía Peruana-Ucayali. Pucallpa. 10 p. (mimeografiado).

SANCHEZ, M.E. 1990. "Evaluación de la biotecnología de recuperación de biomasa a través de la lombriz (Eisenia foetida) en Trópico Húmedo de Pucallpa”. Tesis Ing. Ind. Universidad Nacional Hermilio Valdizán de Huánuco. Huánuco. 80p.

SANCHEZ, P.A. y J.BENITES. 1983. "Opciones Tecnológicas para el manejo racional de suelo en la Selva Peruana. NCSU. CIPA XVI. Est. Exp. Yurimaguas". Serie de separatas 6. Yurimaguas. Serie de separatas 6. Yurimaguas-Perú.

WORTTEN, E. y 5. ALDRICH. 1979. Suelos agrícolas, su conservación y fertilización. 5ta. ed. Edit. Hispano Americana S.A. Mexico. 416 p. 


\section{Grafico 1}

RENDIMIENTO EN PESO FRESCO ( $\mathrm{kg} 10 \mathrm{~m}^{2}$ ) DE PEPINO (Cucumis sativus) POR EFECTO DE HUMUS DE LOMBRICULTURA (HL) PROVENIENTES DE DIFERENTES INSUMOS ORGANICOS, EN UN ULTISOL DEGRADADO DE PUCALLPA-PERÚ, BAJO CONDICIONES DE CAMPO.

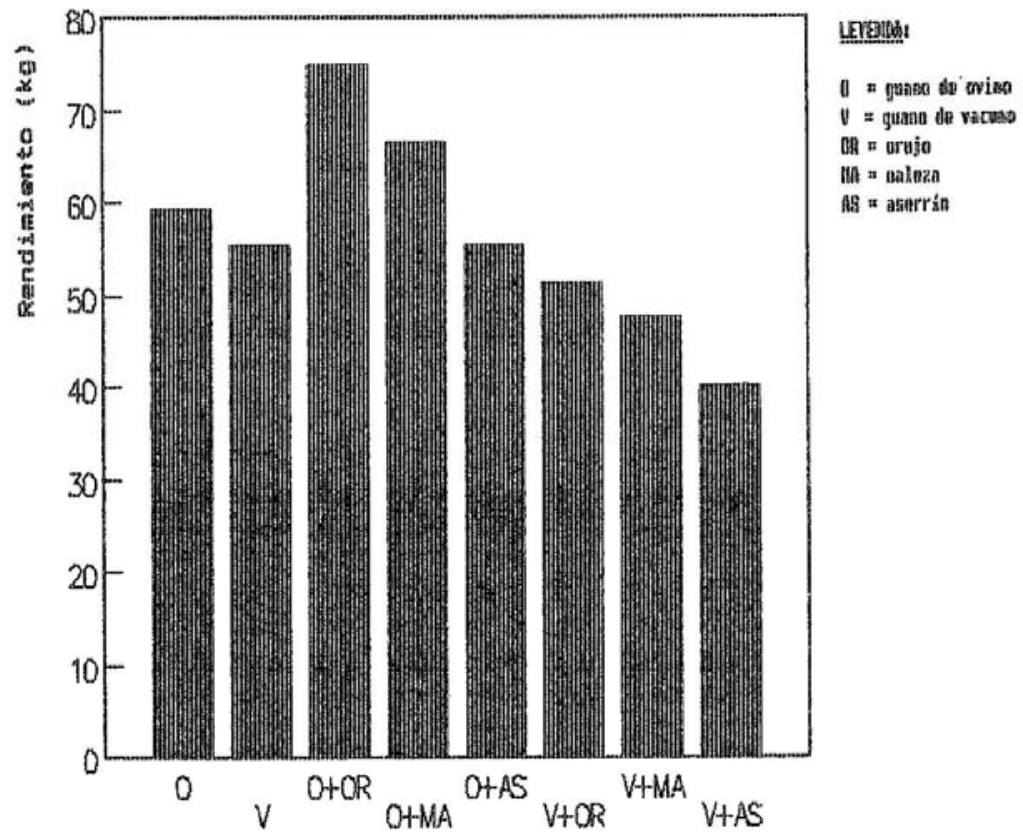

HL. proveniente de diferentess insumos 